\title{
A Capacitance-Based Methodology for the Estimation of Piezoelectric Coefficients of Poled Piezoelectric Materials
}

\author{
Mahmoud Al Ahmad and H. N. Alshareef ${ }^{\mathrm{z}}$
}

Department of Materials Science and Engineering, King Abdullah University of Science and Technology, Thuwal 23955-6900, Kingdom of Saudi Arabia

\begin{abstract}
A methodology is proposed to estimate the piezoelectric coefficients of bulk piezoelectric materials using simple capacitance measurements. The extracted values of $\mathrm{d}_{33}$ and $\mathrm{d}_{31}$ from the capacitance measurements were $506 \mathrm{pC} / \mathrm{N}$ and $247 \mathrm{pC} / \mathrm{N}$, respectively. The $d_{33}$ value is in agreement with that obtained from the Berlincourt method, which gave a $d_{33}$ value of $500 \mathrm{pC} / \mathrm{N}$. In addition, the $d_{31}$ value is in agreement with the value obtained from the optical method, which gave a $d_{31}$ value of $223 \mathrm{pC} / \mathrm{V}$. These results suggest that the proposed method is a viable way to quickly estimate piezoelectric coefficients of bulk unclamped samples.

(c) 2010 The Electrochemical Society. [DOI: 10.1149/1.3494042] All rights reserved.
\end{abstract}

Manuscript submitted August 13, 2010; revised manuscript received September 7, 2010. Published October 4, 2010.

The functionality of piezoelectric materials is being exploited in many integrated applications due to their electrical-mechanical reciprocity. Different methods have been used to measure the piezoelectric coefficients. ${ }^{1-6}$ For these methods, the determination of the variation in physical dimensions in the longitudinal and transversal directions is the key for the measuring the piezoelectric material parameters. For bulk ceramic materials, the most commonly used method to characterize the piezoelectric coefficients is based on using a dynamically varying load, i.e., Berlincourt technique, which offers a rapid and cost effective method of assessing the piezoelectric constants. ${ }^{7}$ The Berlincourt method is based on the direct measurements of force-induced charge

$$
\mathrm{d}_{33}=-\Delta Q / \Delta F
$$

where $Q$ and $F$ are the induced charge and the applied force, respectively. The calibration of the Berlincourt meter is usually carried out using the provided standards.

The resonance technique has also be used to measure the piezoelectric constants and is based on the assumption that samples are infinitely thin or infinitely long, and the corrections for finite dimensions must be taken into account. ${ }^{8}$ The method we report here does not require any expensive equipment and special techniques and the results indicate that can be it can be used for a quick estimation of the piezoelectric constant of bulk ceramics.

\section{Theoretical Background}

The change in piezoceramic materials dimensions due to an external voltage can be detected as a capacitance variation. The capacitance value depends on the material dielectric constant, area, and thickness where, for a parallel-plate configuration, the capacitance of a dielectric film is given by

$$
C=\frac{\epsilon A}{t}
$$

where $\epsilon, A$, and $t$ are the dielectric constant, area, and thickness of the piezoceramic material sandwiched between the capacitor electrodes, respectively. For a poled piezoelectric material, the application of a dc field will result in the expansion or contraction of the piezoelectric element, depending on the direction of the applied dc field relative to the original poling field direction. ${ }^{10}$ The relative variations in material dimensions due to the deformation are correlated with its longitudinal $d_{33}$ and transverse $d_{31}$ piezoelectric constants by the following

$$
\Delta t / t=\mathrm{d}_{33} \mathrm{E}
$$

and

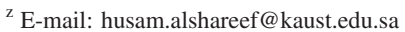

$$
\pm \frac{\Delta A}{A}= \pm 2 \mathrm{~d}_{31} \mathrm{E}+\mathrm{d}_{31}^{2} \mathrm{E}^{2}
$$

where $\Delta A$ and $\Delta t$ are the change in area of the solid and its thickness, respectively. In bulk piezoceramic, the relative change in the material dimensions is the same in both horizontal and vertical directions, i.e., $\mathrm{d}_{33}=2 \mathrm{~d}_{31} \cdot{ }^{10}$ The measured capacitance can therefore increase (if the piezoelectric contracts) or decrease (if the piezoelectric expands). The expansion or contraction of the piezoelectric will of course be determined by the relative direction of the applied filed $\left(E_{a}\right)$ during capacitance measurement compared to the original field $\left(E_{p}\right)$ used to pole the piezoelectric elements. It should be noted that $\mathrm{E}_{\mathrm{a}} \ll \mathrm{E}_{\mathrm{p}}$ in this setup. To distinguish the measured capacitance in these two cases, we define parallel $\left(C_{\downarrow \downarrow}\right)$ and antiparallel capacitances $\left(C_{\mid \uparrow}\right)$, which are determined by the direction of the applied field relative to the original poling field. These parallel and antiparallel capacitances can be given by, respectively

$$
C_{\downarrow \downarrow}=\frac{\epsilon(V)(A-\Delta A)}{(t+\Delta t)}
$$

and

$$
C_{\downarrow \uparrow}=\frac{\epsilon(V)(A+\Delta A)}{(t-\Delta t)}
$$

where $\epsilon(V)$ is the dielectric constant voltage-dependency. Dividing Eq. 5 over Eq. 6 yields a capacitance ratio of

$$
C_{r}=\frac{\left(1-\mathrm{d}_{31} \mathrm{E}\right)^{2}\left(1-\mathrm{d}_{33} \mathrm{E}\right)}{\left(1+\mathrm{d}_{31} \mathrm{E}\right)^{2}\left(1+\mathrm{d}_{33} \mathrm{E}\right)}
$$

The approximation made in Eq. 7 is that the dielectric constant dependence on voltage is the same for parallel and antiparallel arrangement. The validity of this approximation comes from the fact that the capacitance change due to the sample dimension change of the ceramic is significantly larger than the capacitance change due to the domain wall contribution (depoling), especially in the limit that $\mathrm{E}_{\mathrm{a}} \ll \mathrm{E}_{\mathrm{p}}$. The validity of this assumption will be discussed in detail in subsequent section.

Taking into consideration that: $1 \gg \mathrm{d}_{31} \mathrm{E}$; solving for $\Delta A$ and $\Delta t$ yields

$$
\begin{gathered}
\Delta t / t=\left(\frac{1-\sqrt{C_{r}}}{1+\sqrt{C_{r}}}\right) \\
\Delta A / A=\frac{1}{4}\left(\frac{1-\sqrt{C_{r}}}{1+\sqrt{C_{r}}}\right)^{2}+\left(\frac{1-\sqrt{C_{r}}}{1+\sqrt{C_{r}}}\right)
\end{gathered}
$$

where $C_{r}=C_{\downarrow \uparrow} / C_{\downarrow \downarrow}$. It is worth noting that the maximum value for $C_{r}$ is 1.0 and it occurs at $0 \mathrm{~V}$. At $0 \mathrm{~V}$, there is no change either in the material dimensions or parameters, i.e., $\Delta A$ and $\Delta t$ are zero. 


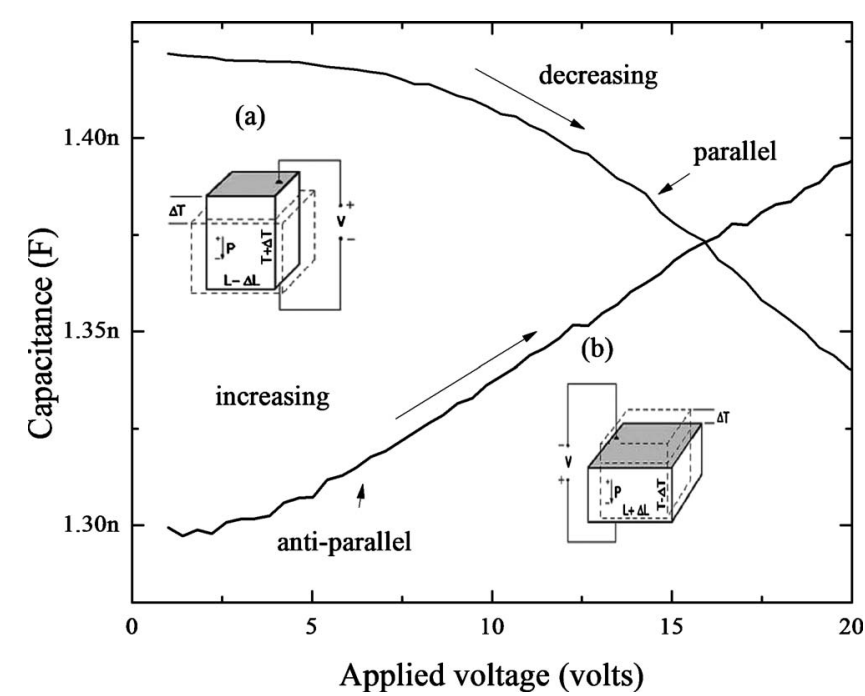

Figure 1. Capacitance measurements: for a positive voltage $(V)$ applied parallel to the poling direction, where the capacitor expands in the $z$-direction but contracts in the $x$ - and $y$-directions as shown in inset (a); if the voltage is reversed, the thickness contracts in the $z$-direction and expands in the $x$ - and $y$-directions, as shown in inset (b). The dimensions of the samples used are $7 \mathrm{~mm}$ length, $1 \mathrm{~mm}$ width, and $0.24 \mathrm{~mm}$ thickness.

\section{Measurements and Analysis}

A parallel-plate capacitor, composed of PZT bulk ceramics of $0.24 \mathrm{~mm}$ thickness has been used in this work. ${ }^{11}$ The ceramic was $1 \mathrm{~mm}$ wide and $7 \mathrm{~mm}$ long. Poling was performed at room temperature using $0.6 \mathrm{kV}$ applied across the $0.24 \mathrm{~mm}$ thickness for $20 \mathrm{~min}$ (i.e., a poling field of $2.5 \mathrm{kV} / \mathrm{mm}$ ). The capacitance was measured with an HP4294A impedance analyzer from -20 to $+20 \mathrm{~V}$ at a frequency of $1 \mathrm{MHz}$. Careful calibration of the impedance analyzer has been carried out using the short-open-load technique. As Fig. 1 shows, the capacitance displays a stable behavior. The variation in capacitance value upon reversal of the sign of the bias voltage is pronounced. Applying a dc stress parallel to the poling direction translates into a decrease in capacitance consistent with an expansion of the material. In contrast, applying a dc stress antiparallel to the poling direction results in an increase in the capacitance, consistent with a contraction of the material.

The change in the capacitance as a function of the voltage can result from two main reasons: one is the change in the dielectric constant due to domain wall motion due to the ferroelectric nature of this material and the second is from the change in the dimensions of the poled material (extension or contraction). We contend that the capacitance change due to the change in physical dimensions is the dominant factor in effecting the observed capacitance change. The reason for this is that the bias applied in measuring capacitance is $20 \mathrm{~V}$, while the poling voltage was $0.6 \mathrm{kV}$. This means that the amount of polarization vector reversal should be small and in fact can be estimated as follows

$$
\Delta C=A \Delta P / \Delta V
$$

From Fig. 2, it can be seen that the change in the polarization due to the change in electric field over the $\pm 20 \mathrm{~V}$ bias applied during capacitance measurement $(20 \mathrm{~V}$ is equivalent to $0.166 \mathrm{kV} / \mathrm{mm}$ for this sample) is equal to $1.6 \mu \mathrm{C} / \mathrm{cm}^{2}$. Using Eq. 10 the corresponding capacitance change from this change in polarization is calculated to be $\Delta C=0.6 \mathrm{pF}$. By comparing this change in capacitance (resulting from ferroelectric polarization reversal) with the total capacitance change in Fig. 1, it is clear that this only amounts to about $1 \%$ of the measured capacitance change. Hence, in this bulk material, it can be concluded that the contraction/expansion in physical dimensions of

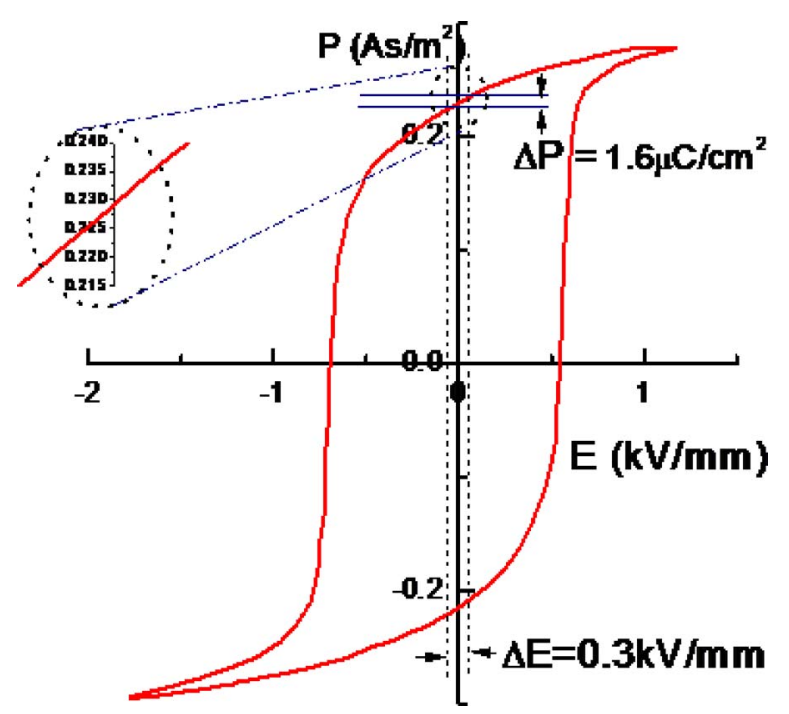

Figure 2. (Color online) Measured polarization curve of the involved piezoelectric material. The maximum polarization is $0.293 \mathrm{C} / \mathrm{m}^{2}$, electric coercive field of -0.686 and $0.556 \mathrm{kV} / \mathrm{mm}$

the sample via the piezoelectric effect is the main contributor to the measured change in capacitance.

Now, the $\triangle A / A$ is computed using Eq. 9. The extracted values $\Delta A / A$ from measurements are shown in Fig. 3 along with their fittings. The fitting equation is found to be

$$
\Delta A / A=-0.024+488 \times 10^{-6} \mathrm{E}+6.1947 \times 10^{-8} \mathrm{E}^{2}
$$

Comparing Eq. 11 with Eq. 4, the first term counts for the spontaneous strain, while the second and the third terms of Eq. 11 account for the piezoelectric effect due to applied voltage, i.e., longitudinal and transversal piezoelectric coefficients, respectively. We find that the extracted values for $d_{33}$ and $d_{31}$ from our capacitance measurements are $506 \mathrm{pC} / \mathrm{N}$ and $247 \mathrm{pC} / \mathrm{N}$, respectively.

To corroborate the results of the $\mathrm{d}_{33}$ and $\mathrm{d}_{31}$ measurements on these samples using the proposed capacitance approach, we have independently measured the piezoelectric constants using alternate established methods. First, the sample under test was characterized using the Berlincourt meter and the measured values of $\mathrm{d}_{33}$ and $\mathrm{d}_{31}$ were 500 and $230 \mathrm{pC} / \mathrm{N}$, respectively, suggesting that the capacitance method gives numbers that are not too far from the actual value (we note that the close match of the $d_{33}$ constant, 500 versus 506 , is probably a coincidence and we are not claiming the method

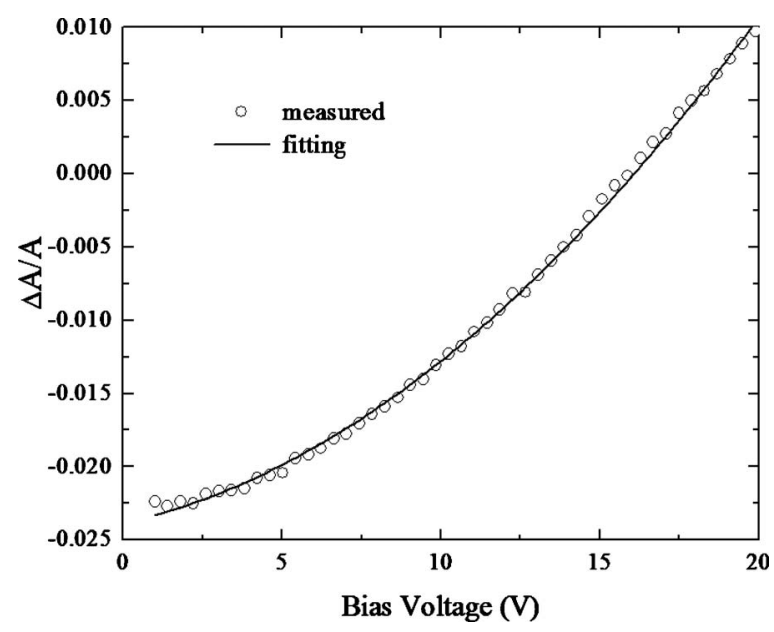

Figure 3. Extracted $\Delta A / A$ values vs dc bias along with the fitting curve. 


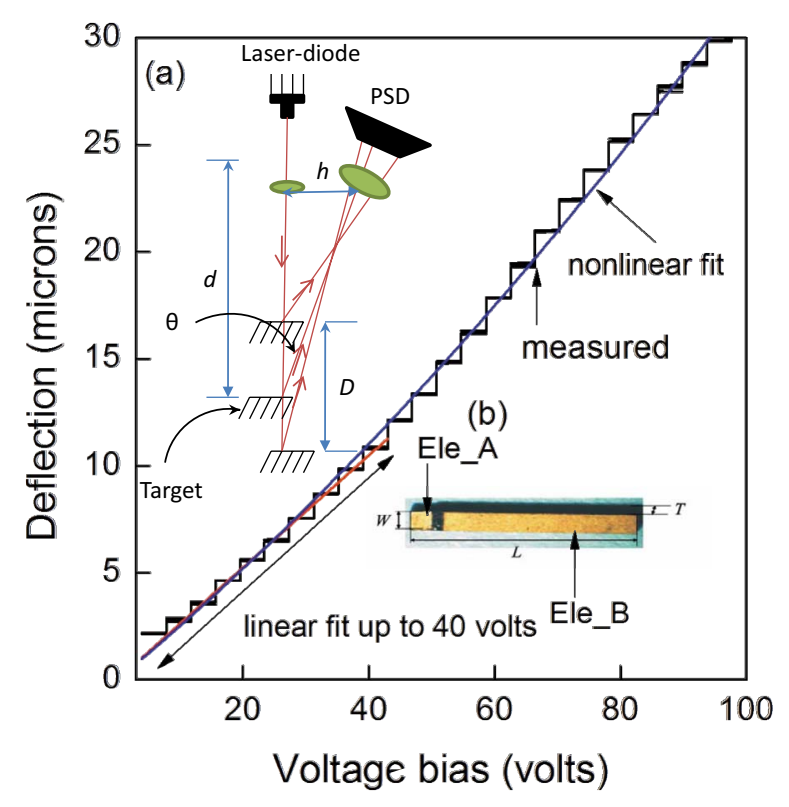

Figure 4. (Color online) Characterization of the piezoelectric actuator deflection. Inset (a): Triangulation calculation. Inset (b) shows the actual sample under test. The capacitance measurements have been performed between electrode (Ele_A) and electrode (Ele_B).

to be universally that accurate). The main point is that is the capacitance method can give a good approximation of the piezoelectric constants. In addition, double-beam interferometry was then used to measure the bending of a cantilever made from the same material as the one used for capacitance measurement. A laser source was used to create a light spot on the cantilever tip which is then observed by a position sensitive device [(PSD) photodiode array] under an oblique angle (Fig. 4). The PSD element supplies a position dependent, analog output voltage proportional to the geometric shift. A diffuse reflection is necessary for the triangulation principle. A calibration is done first to see if there is a target in the measuring range and if the amount of light is sufficient for the PSD. The measurement accuracy can be affected by the angular relationship of the sensor to the target surface.

The distance between the laser source and the PSD detector is $h$. $\mathrm{d}$ represents the reference distance that result from the calibration. From the self-test, both $\mathrm{d}_{0}$ and $\theta_{0}$ are known for the initial state. A deflection, $D$, of the cantilever causes $\mathrm{d}_{0}$ and $\theta_{0}$ to change into $\mathrm{d}$ and $\theta$, from which $D$ can be calculated according to

$$
D=\frac{h \sin \left(\theta-\theta_{0}\right)}{\sin (\theta) h \sin \left(\theta_{0}\right)}
$$

where positive values correspond to upward, negative to downward deflection.

The measured deflection of the cantilever using the beam tracing technique is depicted in Fig. 4. The deflection has been fitted with both linear and nonlinear fitting up to 40 and $100 \mathrm{~V}$, respectively, as shown in Fig. 4. The fitting equations are

$$
\begin{gathered}
y_{\text {linear }}=0.262 \mathrm{~V} \\
y_{\text {nonlinear }}=0.243 \mathrm{~V}+0.00079 \mathrm{~V}^{2}
\end{gathered}
$$

The deflection of the cantilever has been measured at its tip. The deflection is modeled as

$$
\delta=\frac{3}{2} \mathrm{~d}_{31}\left(\frac{L}{T}\right)^{2} \mathrm{~V}
$$

Hence $\mathrm{d}_{31}=0.262 \times \frac{2}{3}(T / L)^{2}(\mu \mathrm{m} / \mathrm{V})$, which produces $\mathrm{d}_{31}$ of $223 \mathrm{pC} / \mathrm{V}$.

Another method that has been used to estimate the piezoelectric constants of piezoelectric ceramics is based on the ferroelectric polarization, permittivity, and electrostriction coefficient as shown in Eq. 16. For our sample, the ferroelectric polarization and dielectric constant are readily available. If we assume electrostrictive coefficients for materials with comparable compositions $\left(\mathrm{Q}_{12}\right)$ then the piezoelectric constant can be estimated as follows ${ }^{12,13}$

$$
\mathrm{d}_{33}=2 \varepsilon_{0} \varepsilon_{r} \mathrm{Q}_{12} \mathrm{P}_{r}
$$

where $\mathrm{Q}_{12}$ is the electrostrictive constant and $\mathrm{P}_{\mathrm{r}}$, the remnant polarization. The dielectric constant of PZT is determined from the equation of a parallel-plate capacitor and is found to be 3500 . The remnant polarization is $23.2 \mu \mathrm{C} / \mathrm{cm}^{2}$, the electrostrictive coefficient is $-0.0315 \mathrm{~m}^{4} / \mathrm{C}^{2}{ }^{11}$ The calculated piezoelectric constant $\mathrm{d}_{33}$ is $455 \mathrm{pC} / \mathrm{N}$. This also gives a reasonable estimate, but requires more measurements than our proposed methodology.

\section{Conclusion}

A simple capacitance-based methodology has been proposed to estimate the piezoelectric constants $\left(\mathrm{d}_{33}\right.$ and $\left.\mathrm{d}_{31}\right)$ of poled and unclamped piezoelectric samples. The estimated constants are close to those obtained by more elaborate techniques such as the Berlincourt and optical methods.

King Abdullah University of Science and Technology assisted in meeting the publication costs of this article.

\section{References}

1. D. Royer and V. Kmetik, Electron. Lett., 28, 1828 (1992).

2. P. Muralt, J. Micromech. Microeng., 10, 136146 (2000).

3. J. F. Shepard, P. J. Moses, and S. Trolier-McKinstry, Sens. Actuators, A, 71, 1338 (1998).

4. D.-G. Kim and H.-G. Kim, in International Symposium on the Application of Ferroelectric 98; pp. 65-68 (1998).

5. M. Al Ahmad, and R. Plana, IEEE Microw. Wirel. Compon. Lett. 19, 140 (2009).

6. B. Gautier, S. Ballandras, V. Blondeau-Patissier, W. Daniau, D. Hauden, and J. C. Labrune, in International Symposium on the Application of Ferroelectric 2002, pp. 99-102 (2002).

7. J. Zelenka, Piezoelectric Resonators and Their Applications, Elsevier Science \& Technology, Amsterdam (1986).

8. Y. Zhang, Z. Wang, and J. D. N. Cheeke, IEEE Trans. Ultrason. Ferroelectr. Freq. Control, 50, 321 (2003).

9. D. M. Pozar, Microwave Engineering, 3rd ed., John Wiley \& Sons, New York (2005).

10. J. E. A. Southin, S. A. Wilson, D. A. Schmitt, and R. W. Whatmore, J. Phys. D: Appl. Phys., 34, 1456 (2001).

11. Vibrit 1334, Argillon GmbH, www.piezo-power.com, last accessed September 25, 2010.

12. T. Abe and M. L. Reed, in IEEE Process, Micro-Electro-Mechanical Systems, pp. 164-169 (1994).

13. K. R. Udayakumar, S. F. Bart, A. M. Flynn, J. Chen, L. S. Tavrow, L. E. Cross, R. A. Brooks, and D. J. Ehrlich, in IEEE Process, Micro-Electro-Mechanical Systems, pp. 109-113 (1991). 